\title{
Sebastian KEMPGEN
}

Slavische Sprachwissenschaft, Universität Bamberg (Deutschland, Bamberg) sebastian.kempgen@uni-bamberg.de

\section{Ein neuer Balkanismus (im Bereich der Onomastik)}

1. „Balkanismen“, also die - teils erworbenen, teils ererbten - Gemeinsamkeiten der Balkansprachen, konstituieren als Merkmale das, was man nach Roman Jakobson [1931] und Nikolaj Trubetzkoy [1931] den Balkansprachbund nennt. Er ist der klassische Anwendungsfall dieser Konzeption einer Angleichung von Sprachmerkmalen geblieben, auch wenn es vielfach unternommen worden ist, diesen Ansatz auf andere Sprachareale zu übertragen. Die Kerngruppe der Balkanismen besteht vornehmlich aus phonologischen und morphologischen Eigenschaften des Sprachsystems (,...weist einen Artikel auf“; ,...verfügt über einen Vokal ähnlich dem Schwa-Laut"), z.T. aus morphonologischen und morphosyntaktischen Eigenschaften („Verdoppelung des Objektes“). Bei einem einzelnen Merkmal ist es typischerweise die Aufgabe, sein Vorkommen unter den Balkansprachen zu beschreiben und seine Genese aus einer dieser Sprachen zu klären und damit zu erklären. Dabei hat die bisherige Forschung zu der Erkenntnis geführt, daß Balkanismen als Bündel von Merkmalen keineswegs in allen Balkansprachen (Griechisch, Bulgarisch, Makedonisch, Albanisch, Rumänisch, Südserbische Dialekte) notwendigerweise gleichermaßen vertreten sind oder vertreten sein müssen: die areale Verbreitung der Merkmale ist nicht zwangsläufig deckungsgleich. Für den Grundgedanken eines Balkanismus reicht es, wenn die bekannten Kriterien erfüllt sind: daß es sich a) in einer der betrachteten Sprachen um eine durch Kontakt erworbene Eigenschaft handelt (Beispiel: Artikel im Bulgarischen und Makedonischen), und daß es sich b) bei den Sprachen, die dieses Merkmal aufweisen, um Angehörige mindestens zweier Sprachfamilien handelt (Beispiel: Artikel u.a. in südslawischen Sprachen und im Griechischen). Damit eignen sich Balkanismen jedoch nicht, wie leicht zu erkennen ist, um jeden einzelnen von ihnen als notwendiges Merkmal der Zugehörigkeit einer Sprache zum Balkansprachbund zu betrachten. Logisch gesehen, ist es vielmehr hinreichend, wenn jeder einzelne Kandidat mindestens einen Balkanismus aufweist. Weist eine Sprache viele Balkanismen auf, so gehört sie zum Kernareal, weist sie weniger solcher Merkmale auf, wird man sie zur Peripherie rechnen. Das Konzept des Sprachbundes nimmt also einen stochastischen Charakter an, eine ganzheitliche Darstellung wäre mit entsprechenden Methoden zu leisten ${ }^{1}$.

2. Die vielleicht spannendste Weiterentwicklung der Sprachbundkonzeption hat dazu geführt, daß man - mindestens für den Balkan — zu der Erkenntnis gelangt ist, daß die Gemeinsamkeiten in diesem Falle keineswegs auf die sprachliche Ebene begrenzt sind, sondern sich im Bereich der materiellen wie immateriellen

${ }^{1}$ Neben den klassischen Arbeiten aus dem Prager Zirkel sei hier auf [Becker 1948; Schaller 1976; Miklas 1982; Hetzer 1989; Asenova 1989; Hinrichs 1993; Reiter 1994; Nikolaeva 1996; Sawicka 1997; Anreiter 1999; Nekula 2003] und natürlich die Bibliographie von Schaller [1977] sowie das Handbuch von Hinrichs [1999] verwiesen. 
Kultur fortsetzen (vgl. [Hirmstedt-Vaid 2012]). So kann man z.B. eine gewisse gemeinsame Balkanarchitektur, die unter osmanischem Einfluß entstanden ist, erkennen, auch wenn sie lokale Besonderheiten aufweist, Speisen und Getränke ähneln sich [Steinke 1990; Burkhart 1991; Blaszczyk/Rohdewald 2018], aber auch Trachten und Kleidung lassen sich untersuchen [Schubert 1993], dazu die bekannten Kopf-Gesten (Bejahung, Verneinung), immaterielles Gut wie die Zuordnung von 'gut' und 'böse' zu links und rechts [van Leeuwen-Turnovcová 1990], das 'sprachliche Weltbild' [Civ'jan 1979; 1990], womöglich die Mentalität [Schmidt 2007] usw. Auch hier gilt es aber, ebenfalls wieder die Einsicht zu konstatieren, daß diese Merkmale gleichfalls stochastischer Natur sind, also nicht überall gleichermaßen ausgeprägt sind und auch nicht gleichermaßen ausgeprägt sein müssen, um als solche akzeptiert werden zu können. So ist z.B. die 'typische Balkanarchitektur' nur Nordgriechenland eigen, während z.B. auf den Kykladen traditionell eine andere Architektur vorherrscht.

Im Grunde ist dieser Bereich also nur eine erneute Bekräftigung der Tatsache, daß Sprache ein Teil der menschlichen Kultur ist und gewöhnlich nicht losgelöst von ihr gedacht werden kann, selbst wenn es aus disziplinären Gründen notwendig und wünschenswert sein sollte, zunächst einmal von dieser Einbettung zu abstrahieren und ein Merkmal für sich zu betrachten.

3. Ziel des vorliegenden Beitrages ist es, einen neuen Balkanismus zu präsentieren, und zwar im Bereich der Onomastik, und damit in einem Bereich, der bislang unter den Balkanismen gar nicht oder nur schwach vertreten war. Unser Beitrag basiert auf den Daten eines sehr interessanten Wikipedia-Artikels: „List of most common surnames in Europe“ (Zugriff: 09.08.2020). Er ist — außer in der englischen Wikipedia - nur in der türkischen vorhanden. In diesem Artikel gibt es für knapp 50 Länder - im Kern jeweils eine Tabelle, in der die 10 bzw. 20 häufigsten Nachnamen aufgelistet werden, teils mit Erläuterungen zur Bedeutung bzw. zur Herkunft der Namen. Die meisten, aber nicht alle Tabellen geben den Rang an, etliche außerdem auch die Frequenz oder den relativen Prozentsatz in der jeweils benutzten Quelle. Kurze Kommentare begleiten die Tabellen. Für die vorliegenden Zwecke ist es ohne Belang, daß die hier zusammengestellten Daten unterschiedlichen Quellen entstammen und unterschiedlich ausführlich dargestellt werden; für uns ist nur die sog. Rangreihenfolge wichtig. Zugleich zeigt der vorliegende Beitrag, daß die Wikipedia nicht nur ein Nachschlagewerk sein kann, sondern daß ihre Einträge selbst Forschung inspirieren können.

Übrigens gilt auch im Bereich der Häufigkeit von Familiennamen das gleiche, was man auch bei anderen sprachlichen Eigenschaften, auch Balkanismen, bedenken muß: bestimmte Aussagen werden zwar ,für eine Sprache“ getroffen, man weiß aber sehr wohl, daß man bei Betrachtung von Dialekten und Regionen z.T. andere Ergebnisse bekäme (vgl. z.B. unten unsere Anmerkung zu den Befunden für St. Petersburg im Gegensatz zu den für ganz Rußland gültigen).

4. Große Sprachgemeinschaften verfügen in der Regel über einen Namenswortschatz von mehreren Tausend Vor- und Familiennamen. (Im übrigen gibt es auch 
keine modernen Industriegesellschaften, die nicht irgendeine Form von Namenssystem entwickelt hätten, wobei die Universalie genau in dieser Abhängigkeit zu verstehen ist: komplexe Gesellschaften entwickeln gestufte Namenssysteme, d.h. ein System, das über die bloßen Ruf- oder Vornamen hinausgeht.) Weil das systemische Inventar groß ist, kann man natürlich mit guter Berechtigung danach fragen, welche Relevanz es besitzen soll, nur die 10 oder 20 häufigsten Familiennamen miteinander zu vergleichen. Diese Frage läßt sich mit einem Blick auf die Statistik sehr schnell überzeugend beantworten (und ist ein Aspekt sog. onomatometrischer Methoden). Es ist offenkundig, da $\beta$ das Inventar an Vornamen oder an Familiennamen nicht gleichmäßig ausgeschöpft wird, wie Vornamenmoden oder solche Fälle zeigen, in denen einem ein zuvor nie gehörter Name begegnet. Offensichtlich liegt statistisch gesehen keine Gleichverteilung in der Häufigkeit aller Namen vor. (Übrigens kann es zwar unter Vornamen, aber nicht unter Nachnamen Modeerscheinungen geben, da Nachnamen ja vererbt und nicht willkürlich gewählt werden können. Das System der Familiennamen ist also von Natur aus stabiler als das Vornamenssystem.) Betrachten wir als Beispiel die Häufigkeitsverteilung der Nachnamen in St. Petersburg aus dem Jahr 1995 (Quelle: http://imja.name/familii/familii-peterburga1.shtml; Zugriff 09.08.2020), wobei wir uns hier auf die häufigsten 27 Namen beschränken:

Tab. 1: Häufigkeit von Nachnamen in St. Petersburg

\begin{tabular}{|c|c|c|c|c|}
\hline Rang & Name & Häufigkeit & Summe & Relativ \\
\hline 1 & Ivanov & 24211 & 24211 & $6,70 \%$ \\
\hline 2 & Smirnov & 16284 & 40495 & $11,21 \%$ \\
\hline 3 & Vasil'ev & 12241 & 52736 & $14,60 \%$ \\
\hline 4 & Petrov & 11297 & 64033 & $17,73 \%$ \\
\hline 5 & Kuznecov & 9648 & 73681 & $20,40 \%$ \\
\hline 6 & Fedorov & 8502 & 82183 & $22,76 \%$ \\
\hline 7 & Mixajlov & 8017 & 90200 & $24,98 \%$ \\
\hline 8 & Pavlov & 6897 & 97097 & $26,89 \%$ \\
\hline 9 & Alekseev & 6739 & 103836 & $28,75 \%$ \\
\hline 10 & Sokolov & 6712 & 110548 & $30,61 \%$ \\
\hline 11 & Andreev & 6487 & 117035 & $32,41 \%$ \\
\hline 12 & Semenov & 6445 & 123480 & $34,19 \%$ \\
\hline 13 & Stepanov & 5909 & 129389 & $35,83 \%$ \\
\hline 14 & Nikolaev & 5692 & 135081 & $37,40 \%$ \\
\hline 15 & Egorov & 5618 & 140699 & $38,96 \%$ \\
\hline 16 & Lebedev & 5510 & 146209 & $40,49 \%$ \\
\hline 17 & Grigor'ev & 5463 & 151672 & $42,00 \%$ \\
\hline 18 & Kuz'min & 5023 & 156695 & $43,39 \%$ \\
\hline 19 & Popov & 4883 & 161578 & $44,74 \%$ \\
\hline 20 & Dmitriev & 4583 & 166161 & $46,01 \%$ \\
\hline 21 & Nikitin & 4468 & 170629 & $47,25 \%$ \\
\hline 22 & Volkov & 4456 & 175085 & $48,48 \%$ \\
\hline
\end{tabular}




\begin{tabular}{|c|l|c|c|c|}
\hline 23 & Solov'ev & 4352 & 179437 & $49,69 \%$ \\
\hline $\mathbf{2 4}$ & Morozov & 4222 & 183659 & $\mathbf{5 0 , 8 5 \%}$ \\
\hline 25 & Bogdanov & 4121 & 187780 & $52,00 \%$ \\
\hline 26 & Aleksandrov & 4093 & 191873 & $53,13 \%$ \\
\hline 27 & Sergeev & 4043 & 195916 & $54,25 \%$ \\
\hline$\ldots$ & $\ldots$ & $\ldots$ & $\ldots$ & $\ldots$ \\
\hline & Summe & & 361.143 & $100,00 \%$ \\
\hline
\end{tabular}

In dieser Tabelle findet sich in der ersten Spalte der Rang, in der dritten der absolute Wert des Vorkommens in der Stichprobe, die insgesamt 361.143 Fälle umfaßt. In der vierten Spalte stehen die aufsummierten Werte, in der fünften Spalte die relativen Anteile der Summen an 100\%. (Die Spalten vier und fünf der Tabelle sind von uns hinzugefügt.)

In der obigen Tabelle haben wir in zwei Zeilen Werte markiert: Mit Rang 10, so zeigt die Tabelle, werden in diesem Falle schon $>30 \%$ aller Familiennamensvorkommen abgedeckt, $>50 \%$ werden mit nur 24 verschiedenen Familiennamen erreicht. Insofern hat es seine statistische Berechtigung, wenn sich Darstellungen der häufigsten Familiennamen auf 10 oder 20 Namen beschränken, weil man damit in der Bevölkerung eben bis 50\% der Fälle erfaßt. In graphischer Darstellung lassen sich die obigen Werte so wiedergeben (Fig. 1):
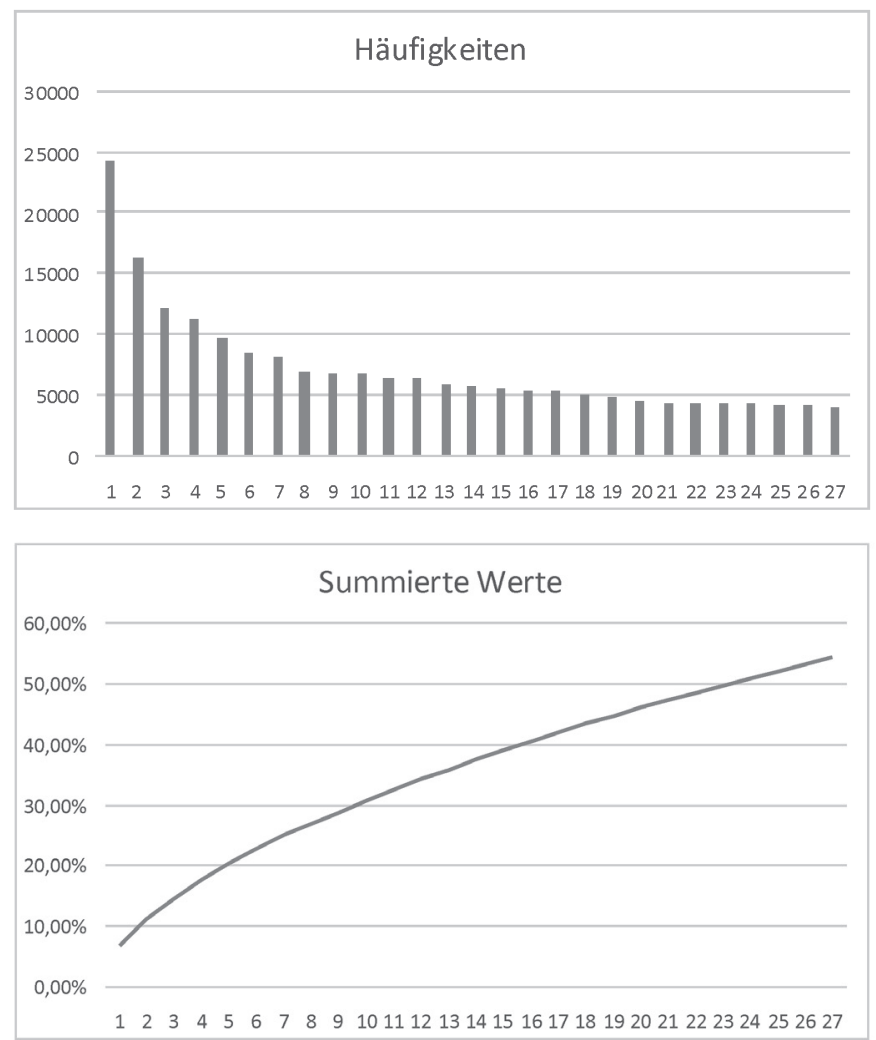

Fig. 1: Absolute und summierte relative Häufigkeiten von Familiennamen (nach Rang) 
Die Darstellung der Häufigkeiten nach der Rangreihenfolge setzt sich natürlich nach rechts noch sehr viel weiter fort, wobei die Häufigkeiten immer weiter relativ gleichförmig abnehmen. In der unteren Darstellung haben wir die beiden Fälle markiert, die wir oben schon angesprochen haben: Mit nur 10 verschiedenen Namen werden $>30 \%$ aller Fälle erfaßt, mit nur 24 verschiedenen Namen werden $>50 \%$ der Fälle erfaßt.

5. Schaut man in dem genannten Wikipedia-Artikel die Tabellen zu den slawischen Sprachen im Hinblick auf die Herkunft der Nachnamen durch, so fällt einem aufmerksamen Leser bald auf, daß sich die einzelnen slawischen Sprachen deutlich unterscheiden, wenn man eine einfache semantisch-etymologische Einteilung in Berufsnamen (Occupational names) - Patronyme (Patronymika) - Übernamen/Beinamen (Agnomina/Cognomina) - Herkunftsnamen/Wohnstättennamen (Toponymika, Oikonymika, Ethnonymika...) etc. verwendet. Dabei kommt es wiederum nicht darauf an, ob diese Klassifikation in jedem Falle eindeutig ist, allen theoretischen Ansprüchen an Klassifikationen genügt usw., da wir letztlich nur den Gegensatz von Patronymika zu allen anderen Namenskategorien verwenden werden. Unsere Beobachtung kann gut eine Gegenüberstellung der Daten für das Serbische mit dem Russischen illustrieren:

Tab. 2: Die 10 häufigsten Familiennamen im Serbischen und im Russischen

\begin{tabular}{|c|c|c|c|c|}
\hline & \multicolumn{2}{|r|}{ Serbia } & \multicolumn{2}{|r|}{ Russia } \\
\hline Rank & Surname & Meaning & Surname & Meaning \\
\hline 1 & Јовановић & son of Jovan (John) & Смирно́в & son of Smirnoy (meek one) \\
\hline 2 & Петровић & son of Petar (Peter) & Ивано́в & son of Ivan \\
\hline 3 & Николић & son of Nikola (Nicholas) & Кузнецо́в & smith's \\
\hline 4 & Марковић & son of Marko (Mark) & Попо́в & priest's son \\
\hline 5 & Ђорђевић & son of Đorđe (George) & Соколо́в & falcon's \\
\hline 6 & Стојановић & son of Stojan (Stoyan) & Ле́бедев & swan's \\
\hline 7 & Илић & son of Ilija (Elijah) & Козло́в & $\begin{array}{l}\text { he-goat's; metaphorically: bearded } \\
\text { one }\end{array}$ \\
\hline 8 & Станковић & son of Stanko (Stanislav) & Но́виков & $\begin{array}{l}\text { newman's; see novik for historical } \\
\text { details }\end{array}$ \\
\hline 9 & Павловић & son of Pavle (Paul) & Моро́зов & frost's \\
\hline 10 & Милошевић & son of Miloš (Miles) & Петро́в & son of Pyotr \\
\hline
\end{tabular}

Sämtliche ersten zehn Plätze in der Rangliste werden im Serbischen von Patronymika eingenommen, während im Russischen nur zwei Patronymika vertreten sind.

In der nachfolgenden Tabelle stellen wir die Daten für alle in dem WikipediaArtikel vertretenen slawischen Sprachen zusammen; die Daten für das Bulgarische und das Makedonische werden dabei extrapoliert. Zusätzlich geben wir die Daten für weitere Balkan-Sprachen an. Für Griechenland bietet der Artikel dabei nur eine summarische Zusammenfassung: "Greek family names are most commonly patro- 
nymics but may also be based on occupation, personal characteristics or location." Als typische Suffixe werden dabei mit regionaler und dialektaler Unterschiedlichkeit v.a. -idis, -akos, -atos, -opoulos, -elis, -oglu verwendet, d.h. neben eigenen Elementen auch ein türkisches. Die Reihenfolge der Einträge haben wir in der Tabelle schon mit einem Farbcode markiert und gruppiert. Dazu verwenden wir eine einfache Skala zur Klassenbildung:

Tab. 3: Patronymika unter den 10 bzw. 20 häufigsten Familiennamen

\begin{tabular}{|l|l|l|l|l|}
\hline (fast) keine & bis ein Viertel & max. die Hälfte & ca. drei Viertel & (fast) alle \\
\hline & & & & \\
\hline
\end{tabular}

\begin{tabular}{|l|c|c|c|c|}
\hline & $/ 10$ & $/ 20$ & andere & Farbcode \\
\hline Serbia & 10 & $?$ & $32 / 38$ & \\
\hline Macedonia & {$[10]$} & $?$ & $18 / 26$ & \\
\hline Bulgaria & {$[10]$} & $?$ & & \\
\hline Montenegro & 8 & $?$ & & \\
\hline Greece & $?$ & $?$ & "The majority of Greek names are patronymic." & \\
\hline Croatia & $2(3)$ & 10 & Serbs & \\
\hline Bosna and & 5 & 11 & & \\
Herzegovina & 5 & 15 & & \\
\hline Romania & 5 & $?$ & & \\
\hline Russia & 2 & 6 & & \\
\hline Belarus & 1 & $?$ & & \\
\hline Poland & 1 & 4 & & \\
\hline Slovakia & 1 & $?$ & & \\
\hline Hungary & 0 & 1 & & \\
\hline Ukraina & 0 & 3 & & \\
\hline Czech Republic & 0 & $?$ & & \\
\hline Slovenia & 0 & $?$ & & \\
\hline Albania & $?$ & $?$ & & \\
\hline
\end{tabular}

6. Im nächsten Schritt sollen die Befunde auf eine Karte übertragen werden (s. Fig. 2). Dabei zeigt sich nunmehr auch in der zweidimensionalen Darstellung deutlich, was die Zahlen der Tabelle bzw. die Gruppierung schon andeuteten: Es ergibt sich eine klare Dominanz von Patronymika unter den 10 bzw. 20 häufigsten Familiennamen auf dem Balkan, und zwar mit einem Kernareal Bulgarien - Makedonien - Serbien, dazu Griechenland und Montenegro; etwas schwächer ist die Tendenz in den peripheren Gebieten des Balkan vertreten: Rumänien, Kroatien, Bosnien. Im zentralen Bereich Europas gibt es einen Gürtel von Ländern, in denen die gleiche Tendenz so nicht existiert: Ukraine, Ungarn, Slowenien, Tschechische 


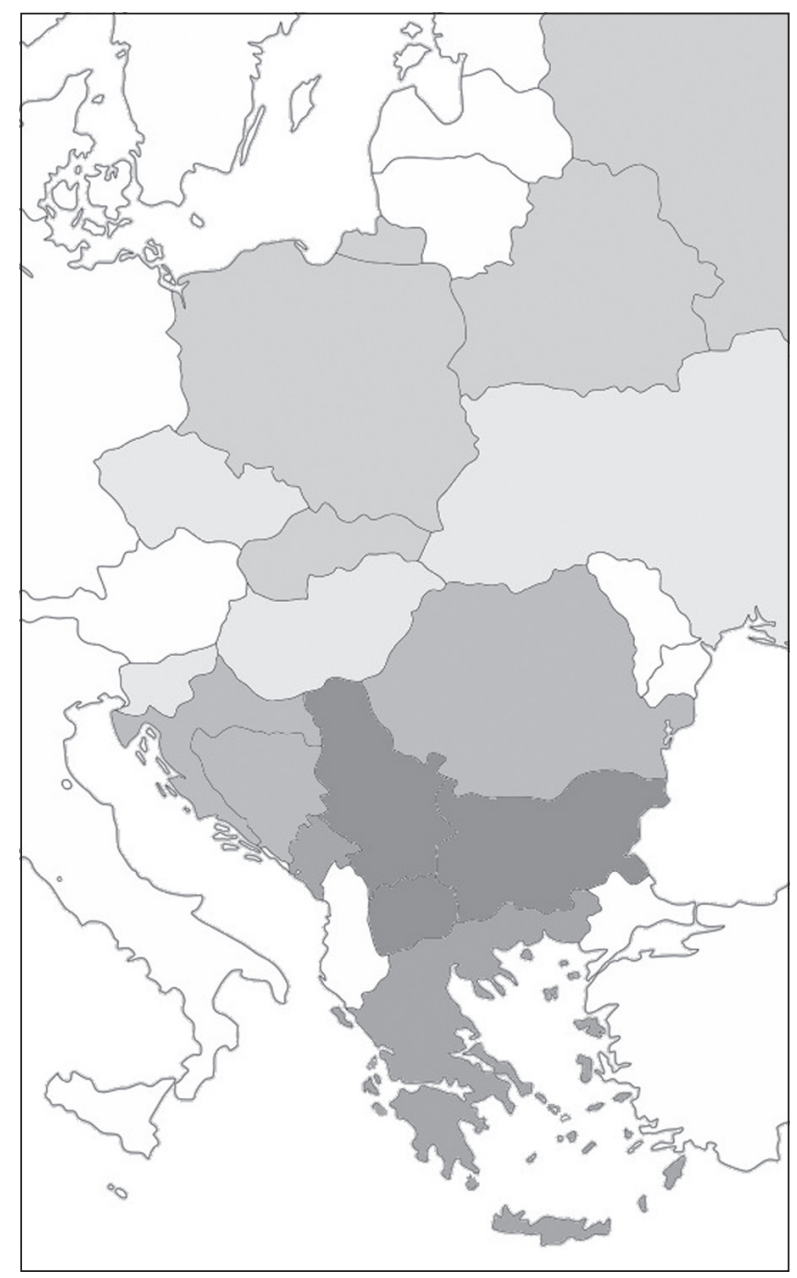

Fig. 2: Anteile der Patronymika in der Slavia und auf dem Balkan

Republik. Etwas stärker wiederum ist sie in der nördlichen Zone vertreten, die Rußland, Belarus, Polen und die Slowakei umfaßt. Dies also, um es noch einmal deutlich zu konstatieren, ist der neue (onomastische) Balkanismus, der hier vorgestellt werden sollte: eine klare oder überwältigende Dominanz der Patronymika unter den 10 bzw. 20 häufigsten Familiennamen, die, wie oben gezeigt, leicht $50 \%$ aller Vorkommen ausmachen. Die Areale der verschiedenen Intensitäten dieser Erscheinung fällt dabei weitgehend mit den Bereichen zusammen, die man auch aus anderen sprachlichen Erscheinungen kennt, und die natürlich mit der Dauer und Intensität der osmanischen Herrschaft auf dem Balkan korrelieren. Wenn die genannte Erscheinung typisch für den Balkan ist, so bedeutet das natürlich im Umkehrschluß nicht, daß sie anderswo nicht vorhanden sein könnte bzw. dürfte. So fällt z.B. auf, daß die Angaben zu St. Petersburg oben von den Angaben für ganz Rußland deutlich abweichen: in St. Petersburg haben wir ebenfalls schon unter den häufigsten 10 Familiennamen viele Patronymika (nämlich 7). 
7. Die Ergebnisse bedürfen natürlich eines Kommentars und einer Erklärung. Wie bei den klassischen Balkanismen ist die Dominanz der Patronymika ein erworbenes Merkmal, kein ererbtes. Es sind mehr als zwei Sprachfamilien (oder Zweige) einbezogen. Das Altgriechische, als älteste bezeugte Sprache des Areals, kannte schon Beinamen im allgemeinen Sinne, etwa „Mann aus X“, „Sohn des X“ usw. Das Neugriechische hat die Eigenschaft also nur beibehalten, nicht erworben. Die modernen Familiennamen sind allgemein erst im Mittelalter entstanden. Deshalb erlauben die Balkanländer mit ihrer Dominanz der Patronymika interessanterweise einen Blick zurück in die Geschichte: wenn heute Patronymika dominieren, so bedeutet das ja, daß die betreffenden Vornamen im Mittelalter dominant waren: Jovan, Petar, Nikola, Marko, Đorđe, Stojan, Ilija, Stanko, Pavle, Miloš etc. in Serbien beispielsweise.

Der Grund, warum sich die Patronymika auf dem Balkan so durchgesetzt haben, dürfte in der gemeinsamen Wirtschaftsgeschichte der Region liegen, also im außersprachlichen Bereich: Hirten wurden und werden kaum nach ihrem Beruf bezeichnet, weil der nicht besonders distinktiv ist und nicht „,vor Ort“ im Dorf ausgeübt wird ${ }^{2}$. Als eigenständige Tätigkeiten fand man in den Balkandörfern offenbar einen Popen und einen Schmied - daher als Familiennamen mit einiger Häufigkeit noch Popović, Popovski etc. und Kovač, Kovačević etc. Im Russischen ist das übrigens nicht anders: Kuznecov ist die Nr. 3 der Liste, Popov die Nr. 4. Andere Berufe kommen unter den 20 häufigsten Nachnamen nicht vor. Nur dort also, wo die Arbeitsteilung zu der Zeit, als sich die Nachnamen etablierten, schon weiter fortgeschritten war und eine größere Bevölkerungszahl und/oder eine Städtebildung Handwerke sich herausbilden ließen, finden wir heute mit großer Häufigkeit entsprechende Familiennamen. In dieser Hinsicht sind übrigens die Daten zur Ukraine sehr interessant (auch im Gegensatz zu Rußland), denn hier kommen sehr häufig vor: Melnyk, Ševčenko, Kovalenko/Koval, Bondarenko/Bondar, Tkačenko/Tkačuk, Kovalčuk, Kravčenko, Oliynyk - 11 Fälle von 20, dabei dominierend vor allem unter den ersten 10 (9 von 10). Daß gerade im russischen Raum bei der Betrachtung des Namenssystems und seiner Zusammensetzung auch berücksichtigt werden muß, daß ja der Vatersname ebenfalls existierte, ist offensichtlich, gilt aber für andere Länder auch.

\section{LITERATURVERZEICHNIS}

Anreiter P. Der Balkansprachbund. // Sprachen in Europa. Sprachsituation und Sprachpolitik in europäischen Ländern (Innsbrucker Beiträge zur Kulturwissenschaft. Bd. 30) / hrsg. v. I. Ohnheiser et al. Innsbruck: Inst. f. Sprachwissenschaft, 1999. P. 297-318.

Asenova P. Balkansko ezikoznanie. Osnovni problemi na balkanskija ezikov sajuz. Sofia: Nauko i izkustvo, 1989 bzw. Veliko Tărnovo: Faber, 2002 (2. ed.). 375 pp.

Blaszczyk A., Rohdewald St. (eds.) From Kebab to Ćevapčići. Foodways in (Post-)Ottoman Europe. (Interdisziplinäre Studien zum östlichen Europa, 6.) Wiesbaden: Harrassowitz, 2018. 390 pp.

Burkhart D. (Hg.) Körper, Essen und Trinken im Kulturverständnis der Balkanvölker. Beiträge zur Tagung vom 19.-24.11.1989 in Hamburg. (Balkanologische Veröffentlichungen, Bd. 19.) Wiesbaden: Harrasowitz, $1991.300 \mathrm{pp}$.

Civ'jan T. V. Kategorija vidimogo/nevidimogo: balkanskie marginalii. // Balcanica. Lingvističeskie issledovanija / otv. red. T. V. Civ'jan. Moskva: Nauka, 1979. S. 201-207.

\footnotetext{
${ }^{2} \mathrm{Zu}$ den Wanderhirten vgl. insbesondere Kahl [2008].
} 
Civ'jan T. V. Lingvističeskie osnovy balkanskoj modeli mira. Moskva: Nauka, 1990. $207 \mathrm{~s}$.

Hetzer A. Der Balkansprachbund als Forschungsproblem. // Südost-Forschungen 1989. 48. P. 177-194.

Himstedt-Vaid P., Hinrichs U., Kahl T. (Hrsg.) Handbuch Balkan. (Slavistische Studienbücher. Neue Folge. Bd. 23.) Wiesbaden: Harrassowitz, 2012. 844 pp.

Hinrichs U. Ziele und Wege der Balkanlinguistik. // Sprache in der Slavia und auf dem Balkan: Slavistische und balkanologische Aufsätze. Norbert Reiter zum 65. Geburtstag / Hrsg. U. Hinrichs, H. Jachnow, R. Lauer, G. Schubert. (Opera Slavica. Neue Folge. Bd. 25.) Wiesbaden: Harrassowitz 1993. P. $101-115$.

Hinrichs $U$. (Hg) Handbuch der Südosteuropa-Linguistik. (Slavistische Studienbücher. Neue Folge. Bd. 10.) Wiesbaden: Harrassowitz, 1999. 1079 pp.

Jakobson R. Über die phonologischen Sprachbünde // TCLP. 1931. No. 4. P. 234-240.

Kahl T. Hirten in Kontakt. Sprach- und Kulturwandel ehemaliger Wanderhirten (Albanisch, Aromunisch, Griechisch). (Balkanologie. Beiträge zur Sprach- und Kulturwissenschaft, Bd. 2.) Münster: LIT, 2008. 348 pp.

van Leeuwen-Turnovcová J. Rechts und links in Europa. Ein Beitrag zur Semantik und Symbolik der Geschlechterpolarität. (Balkanologische Veröffentlichungen. Bd. 16.) Wiesbaden: Harrassowitz, 1990. $280 \mathrm{pp}$.

Miklas H. Trubetzkoys Sprachbundbegriff aus der Sicht der jüngeren Forschung. // Festschrift für Wilhelm Lettenbauer zum 75. Geburtstag / hrsg. v. A. Měšt'jan, E. Weiher. Freiburg: U. W. Weiher, 1982. P. 131-154.

Nikolaeva T. M. Prosodija Balkan. Slovo - vyskazyvanie - tekst. Moskva: Indrik 1996. 350 pp.

Reiter N. Grundzüge der Balkanologie. Ein Schritt in die Eurolinguistik. (Balkanologische Veröffentlichungen des Osteuropa-Institutes. Bd. 22.). Berlin: Harrassowitz, 1994. X, 699 pp.

Schaller H. W. Zur heutigen Auffassung des Begriffs „Sprachbund“ // Die Welt der Slaven 1976. 21/1. P. 208-216.

Schaller H. W. Bibliographie zur Balkanphilologie. Heidelberg: Carl Winter, 1977. $109+$ VII pp.

Schmidt W.-H. Balkanische Mentalität. Zur Konkretisierung einer Fragestellung. // Beitrage zur slawischen Philologie. Festschrift für Fred Otten / Hrsg. v. W. Gladrow und D. Stern. (Berliner Slawistische Arbeiten. Bd. 31.) Frankfurt a. Main: Peter Lang 2007. P. 257-272.

Schubert G. Kleidung als Zeichen. Kopfbedeckungen im Donau-Balkan-Raum. (Balkanologische Veröffentlichungen. Bd. 20.) Wiesbaden: Harrassowitz, 1993. 642 pp.

Steinke K. Die Türken und die Balkanküche: Kulinarisches und Sprachliches aus Bulgarien und Rumänien. // Südosteuropa-Mitteilungen 1990. 30/2. P. 65-72.

Trubetzkoy N. S. Phonologie und Sprachgeographie. // TCLP 4. S. 228-234. 